\title{
Primary Laryngeal Aspergillosis in an Immunocompetent Host
}

\author{
Kaustuv Das Biswas, ${ }^{1}$ Ankit Choudhary, ${ }^{1}$ Swapan Kumar Ghosh, ${ }^{1}$ Subhradev Biswas ${ }^{1}$
}

\begin{abstract}
Introduction
Aspergillosis of Larynx is very rare and may present with symptoms suspicious of malignancy. Prevention of dissemination warrants early diagnosis. It is found mainly in Immunocompromised patients and is usually necrotizing, invasive with disseminated systemic infection, associated with poor prognosis. In Immunocompetent patients it is extremely rare and may present as colonization associated with excellent prognosis.

Case Report

A 43 year old male patient presented with hoarseness of voice for about 3 months with whitish irregular lesion that involved the anterior 1/3 of both the vocal cords. A cheesy material was found covering indurated lesion of both the vocal cords, intraoperatively. Stripping of mucosa over vocal cords was done and cheesy material collected and sent for HPE which revealed Aspergillus hyphae overlying Mild dysplastic changes. Following diagnosis the patient was followed up with oral dosages of Itraconazole.

Discussion

The primary Aspergillosis of larynx is extremely rare. Involvement is always secondary to immunocompromised states like AIDS, malignancy, Diabetes, etc. This was a rare case in Immunocompetent patient.

Management constitute removal of the vocal cord lesions during biopsy. Amphotericin B is first-line agent for this infection, however aerosolised and liposomal amphotericin B offer effective dosing with less toxicity. Newer antifungal Itraconazole may produce more reliable results.
\end{abstract}

ABSTRACT

Kevwords

Larynx; Aspergillosis; Itraconazole

1 spergillosis of larynx is extremely rare and is almost always secondary to advanced stage of broncho-pulmonary aspergillosis. The diagnosis of aspergillus is important as it mimics the symptoms suspicious of malignancy; ${ }^{1}$ but if the diagnosis is delayed or neglected, the aspergillosis may promulgate extensively leading to necrotising, invasive and severe systemic symptoms with poor prognosis. Even in patients with compromised immunity, opportunistic Aspergillus infection primarily affecting Larynx is remarkably low. The cause of suppression for immunity could be many fold like in the case of AIDS, malignancy, diabetes mellitus, drug induced (steroids, chemotherapeutic agents or antibiotics) or secondary to radiotherapy. ${ }^{2}$ In patients with competent or normal immunity the laryngeal aspergillosis is eminently low and may presents as local colonization initially rather being invasive in nature. Novelty lies in considering the diagnosis of primary disease even in immunocompetent host and treating it aggressively to prevent debilitating complications.

Laryngeal aspergillosis mainly affects vocal cords and is primarily presented with hoarseness of voice and rarely with respiratory obstruction or stridor. ${ }^{3}$ This report primarily presents the clinical manifestations, diagnosis and management for an immunocompetent patient presenting with Aspergillus fumigatus infection of the vocal cords.

Amphotericin B was earlier considered as the drug of choice for management of Laryngeal Aspergillosis but have severe systemic side effects. In our case oral Itraconzole was tried successfully which is relatively

1 - Department of ENT, SSKM hospital and IPGMER, Kolkata.

Corresponding author:

Dr Ankit Choudhary

email: dr.ankitchoudhary@gmail.com 
less toxic but equally effective in treating Laryngeal Aspergillosis, thus shifting the whole paradigm of management to a safer alternative.

\section{Case Report}

A 43 year old male, shopkeeper by occupation presented with complaints of hoarseness of voice for 3 month. There was no associated history of fever, cough, pain or respiratory distress. Indirect Laryngoscopy revealed whitish irregular lesion involving the anterior $1 / 3$ of both vocal cords which were mobile. Other systemic and ENT examination were all within normal limits. Chest $\mathrm{X}$-Ray and blood parameters were all within normal limits. Serology (Hepatitis B,C and HIV) was negative. It was provisionally diagnosed as a case of keratosis of the larynx and the patient underwent Microlaryngeal surgery under general anaesthesia. Intraoperative evaluation revealed indurated lesion over the medial margin and upper surface of anterior one third of both the vocal cords and the anterior commisure. False Cord and Subglottis was free of any lesion. Cheesy material was found to cover the indurated lesion. (Fig.1) Cheesy material was collected and stripping of mucosa was done in affected part and sent for histopathological examination.

Pathology revealed fungal hyphae morphologically resembling aspergillus overlying mild to moderate dysplastic changes (Fig. 2). Blood evaluation including total WBC count, differential count, blood sugar, serology, CD count was all within normal limits. Thorough evaluation of chest also revealed no abnormality. The case was thus diagnosed as a case of Primary aspergillosis of larynx in an immunocompetent host. Patient was then followed up on antifungal (Itraconazole 100mg BD for 2 months). Follow up fibreoptic laryngoscopy (FOL) at 2 month, 6 month (Fig. 3) and 1 year revealed no obvious abnormality.

\section{Discussion}

Actinomycosis, Blastomycosis, Histoplasmosis, Candidiasis, Leptospirosis and Rhinosporidiosis are the common primary fungal infestation of Larynx. ${ }^{4}$ Aspergillosis is caused by the ubiquitous fungi of family Aspergillus (Species A. fumigatus, A. niger, A. flavus). Aspergillosis can broadly be subdivided onto two categories viz. Superficial and Deep. Superficial category involves the mucosal lining where as Deep category involves the deeper tissues and may disseminate by blood to affect the lungs, liver, heart, brain, kidneys, spleen, gastro-intestinal tract and lymph nodes.

As per histopathology, Aspergillosis can be primarily categorized as necrotizing, suppurative and granulomatous or pseudo-membranous varieties. Aspergillus fumigatus and niger are the two most

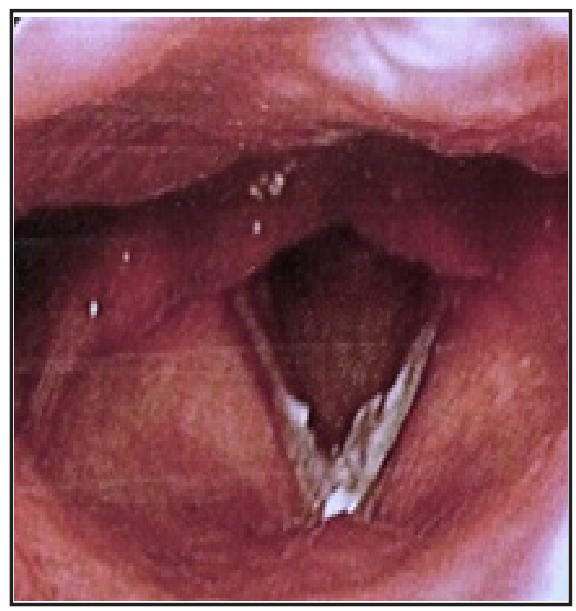

Fig. 1. Pre-operative FOL showing whitish irregular lesion involving the anterior $1 / 3$ of both vocal cords

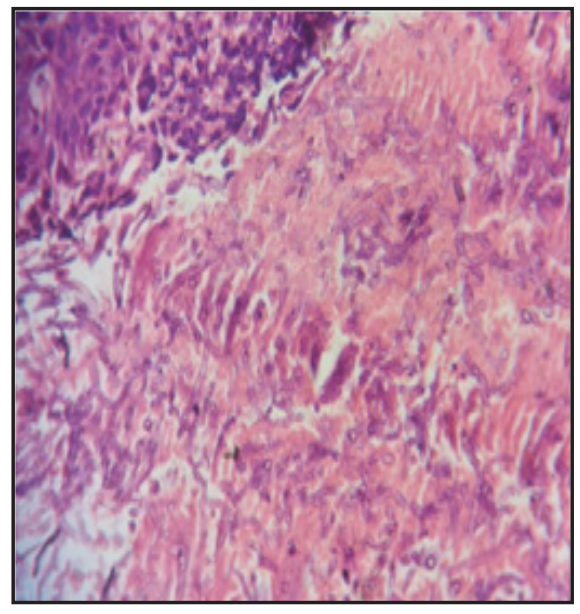

Fig. 2: HPE slide revealing fungal hyphae overlying mild to moderate dysplastic change (H\&E, 10X)

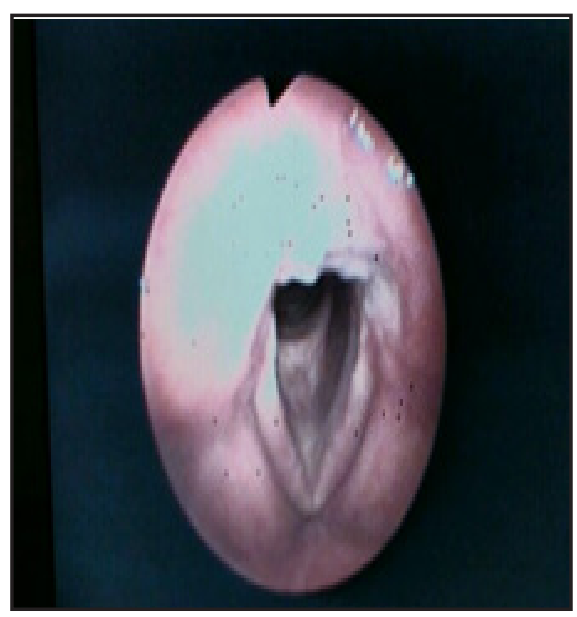

Fig. 3. Post operative FOL showing complete resolution of the lesion 
common pathogenic organism in Human. It is seen more commonly in tropical region. Primary infestation of larynx by Aspergillus is uncommon. Aspergillus Infestation of Larynx is almost always secondary to chronic debilitating diseases like diabetes, malignancy and other immunological diseases. It is often seen in patients receiving long standing immunosuppressive treatment, cytotoxic drugs (cancer chemotherapy) and radiotherapy. However, in this patient, none of the above-mentioned factors was present.

The localized infection of Aspergillus in larynx is extremely rare and is almost always secondary to Aspergillosis of tracheobronchial tree (Commonly Allergic Bronchopulmonary Aspergillosis and Invasive Pulmonary Aspergillosis). Notably, it was observed that none of the 98 patients affected by systemic aspergillosis in study by Young et $\mathrm{al}^{5}$ had laryngeal involvement. The hyphae of Aspergillus stains basophilic with hematoxylin and eosin stain. Gomori Methenamine Silver stain, Reticular silver impregnation method, PAS and Hematoxylin stain, give a better morphological appearance of the hyphae. It is identified by its peculiar circular mycelium with septate hyphae, occasional folds and dichotomous branching. ${ }^{6}$

Early diagnosis of aspergillosis infection is facilitated by the symptoms which mimics that of vocal cord carcinoma (hoarseness of voice). For the patient early relief of symptoms might be facilitated by the removal of vocal cord lesions during the biopsy. It is very important to diagnose the infection in early stage to adequately manage and prevent further dissemination of the disease. Management of Aspergillosis in patients with normal immune system is generally by antifungal agents. However, the effect of antifungal agents in treatment of immuno-compromised patients is not very clear. Amphotericin B is considered as the primary and first-line agent for treating this infection in immunocompromised patients. ${ }^{7}$ Since it is associated with high incidence of toxicity and side effects, Aerosolized and liposomal amphotericin B offer hope of higher and more effective dosing with less toxicity. Newer antifungal agents like Itraconazole are also showing promising results as per recent studies. ${ }^{8}$ The liquid formulation of Itraconazole may produce more reliable and constant circulatory levels but interaction with Rifampicin, Phenytoin, Cyclosporine and other drugs may be a problem.

Primary aspergillosis of Larynx should be considered as a differential diagnosis in patients presenting with hoarseness even in immunocompetent host and should be treated aggressively to prevent any dissemination. Itraconazole was found to be effective antifungal agent with encouraging results in our case.

\section{References}

1. Kheir SM, Flint A, Moss JA. Primary aspergillosis of the larynx simulating carcinoma. Hum Pathol. 1983;14:184-6

2. Doloi PK. Primary Aspergillosis of the Larynx: A Case Report. Indian J Otolaryngol Head Neck Surg. 2014; 66(Suppl 1):326-8

3. Ferlito A. Primary aspergillosis of the larynx. J Laryngol Otol. 1974; 88:1257-63

4. Friedmann I. Otolaryngology by Paparella, M. and Schumrick, D.A., editors. 1973; Philadelphia W.B. Saunders Co. p 616

5. Young RC, Benett JE, Vogel CL, Carbone PP, DeVita VT. Aspergillosis. The spectrum of the disease in 98 patients. Medicine 1970; 49:147-73

6. Sood VP, Daharwal A, Bahadur S. Primary aspergillosis of the larynx. Indian J Otolaryngol Head Neck Surg. 1983; 35:41

7. Benson-Mitchell R, Croft CB, Gallimore A. Aspergillosis of the larynx. J Laryngol Otol. 1994;108:883-5

8. Ran Y, Yang B, Liu S, Dai Y, Pang Z, Fan J, Bai H, Liu S. Primary vocal cord aspergillosis caused by Aspergillus fumigatus and molecular identification of the isolate. Med Mycol. 2008; 46:475-9. 